\title{
SOLVABILITY OF A LINEAR NON-LOCAL BOUNDARY VALUE PROBLEM FOR NONLINEAR FUNCTIONAL DIFFERENTIAL EQUATIONS
}

\author{
ZDENĚK OPLUŠTIL
}

\begin{abstract}
New sufficient conditions are established for the solvability as well as unique solvability of a linear non-local boundary value problem for nonlinear functional differential equations.
\end{abstract}

\section{Introduction and notation}

On the interval $[a, b]$, we consider the functional differential equation

$$
u^{\prime}(t)=F(u)(t),
$$

where $F: C([a, b] ; \mathbb{R}) \rightarrow L([a, b] ; \mathbb{R})$ is a continuous (in general) nonlinear operator. As usual, by a solution of this equation we understand an absolutely continuous function $u:[a, b] \rightarrow \mathbb{R}$ satisfying the equality (11) almost everywhere on the interval $[a, b]$. For the basic theory of functional differential equations we refer to monographs [1, 4, 6, 8] (see also references therein). In spite of many interesting results there is a broad field for further investigations, namely, in the question on the solvability of various boundary value problems for the equation (11).

In the present paper, along with the equation (1), we consider the non-local boundary condition

$$
h(u)=c,
$$

where $h: C([a, b] ; \mathbb{R}) \rightarrow \mathbb{R}$ is a (non-zero) linear bounded functional and $c$ is a real number. The results presented below can be regarded as a generalization of those established in [2] (see also [3, Chapter 14]).

2000 Mathematics Subject Classification: $34 \mathrm{~K} 10$.

Keywords: non-local boundary value problem, nonlinear functional differential equation, existence, uniqueness.

The research was supported by the Ministry of Education, Youth and Sports of the Czech Republic under the project 2E08017 "Procedures and Methods to Increase Number of Researchers". 


\section{ZDENĚK OPLUŠTIL}

The following notation is used in the sequel.

(1) $\mathbb{R}$ is the set of all real numbers. $\mathbb{R}_{+}=[0,+\infty]$.

(2) $C([a, b] ; \mathbb{R})$ is the Banach space of continuous functions $v:[a, b] \rightarrow \mathbb{R}$ with the norm $\|v\|_{C}=\max \{|v(t)|: t \in[a, b]\}$.

(3) $C\left([a, b] ; \mathbb{R}_{+}\right)=\{u \in C([a, b] ; \mathbb{R}): u(t) \geq 0$ for $t \in[a, b]\}$.

(4) $A C([a, b] ; D)$, where $D \subseteq \mathbb{R}$, is the set of absolutely continuous functions $v:[a, b] \rightarrow D$.

(5) $L([a, b] ; \mathbb{R})$ is the Banach space of Lebesgue integrable functions $p:[a, b] \rightarrow$ $\mathbb{R}$ with the norm $\|p\|_{L}=\int_{a}^{b}|p(s)| \mathrm{d} s$.

(6) $L\left([a, b] ; \mathbb{R}_{+}\right)=\{p \in L([a, b] ; \mathbb{R}): p(t) \geq 0$ for almost all $t \in[a, b]\}$.

(7) $\mathcal{L}_{a b}$ is the set of linear operators $\ell: C([a, b] ; \mathbb{R}) \rightarrow L([a, b] ; \mathbb{R})$ for which there is a function $\eta \in L\left([a, b] ; \mathbb{R}_{+}\right)$such that

$|\ell(v)(t)| \leq \eta(t)\|v\|_{C} \quad$ for almost all $t \in[a, b]$ and all $\quad v \in C([a, b] ; \mathbb{R})$.

(8) $P_{a b}$ is the set of operators $\ell \in \mathcal{L}_{a b}$ transforming the set $C\left([a, b] ; \mathbb{R}_{+}\right)$into the set $L\left([a, b] ; \mathbb{R}_{+}\right)$.

(9) $F_{a b}$ is the set of linear bounded functionals $h: C([a, b] ; \mathbb{R}) \rightarrow \mathbb{R}$.

(10) $P F_{a b}$ is the set of functionals $h \in F_{a b}$ transforming the set $C\left([a, b] ; \mathbb{R}_{+}\right)$ into the set $\mathbb{R}_{+}$.

(11) $K\left([a, b] \times \mathbb{R}_{+} ; \mathbb{R}_{+}\right)$is the set of functions $f:[a, b] \times \mathbb{R}_{+} \rightarrow \mathbb{R}_{+}$satisfying the Carathéodory conditions, i.e., $f(\cdot, x):[a, b] \rightarrow \mathbb{R}_{+}$is a measurable function for all $x \in \mathbb{R}_{+}, f(t, \cdot): \mathbb{R}_{+} \rightarrow \mathbb{R}_{+}$is a continuous function for almost all $t \in[a, b]$, and for every $r>0$ there exists $q_{r} \in L\left([a, b] ; \mathbb{R}_{+}\right)$such that

$$
|f(t, x)| \leq q_{r}(t) \text { for almost all } t \in[a, b] \text { and all } x \in[0, r] .
$$

\section{Main results}

First formulate the following definition of a solution of the problem (1), (2).

Definition 2.1. By a solution to the equation (11) we understand an absolutely continuous function $u:[a, b] \rightarrow \mathbb{R}$ satisfying the equality (11) almost everywhere on the interval $[a, b]$. A solution to the equation (11) satisfying the boundary condition (2) is said to be a solution to the problem (11), (2).

We assume in theorems below that the functional $h$ in the boundary condition (2) admits the representation $h=h_{0}-h_{1}$, where $h_{0}, h_{1} \in P F_{a b}$. There is 
no lost of generality in assuming this, because an arbitrary linear bounded functional can be expressed in this form (see [7]). Moreover, the following assumption is used:

(H) $F: C([a, b] ; \mathbb{R}) \rightarrow L([a, b] ; \mathbb{R})$ is a continuous operator such that the relation

$$
\sup \left\{|F(v)(\cdot)|: v \in C([a, b] ; \mathbb{R}),\|v\|_{C} \leq r\right\} \in L\left([a, b] ; \mathbb{R}_{+}\right)
$$

is satisfied for every $r>0$.

Before formulation of the main results we introduce the following notation. Having a point $c \in[a, b]$ and a functional $h_{0} \in P F_{a b}$, for any $\lambda \geq 0$, we put

$$
h_{0, c}^{\lambda}(v) \stackrel{\text { def }}{=} h_{0}(v)-\lambda v(c) \quad \text { for } \quad v \in C([a, b] ; \mathbb{R}) .
$$

Obviously, $h_{0, c}^{0} \in P F_{a b}$. Therefore, we set

$$
\lambda_{c}^{*}=\sup \left\{\lambda \geq 0: h_{0, c}^{\lambda} \in P F_{a b}\right\} .
$$

It is clear that $0 \leq \lambda_{c}^{*} \leq h_{0}(1)$ and

$$
h_{0, c}^{\lambda_{c}^{*}} \in P F_{a b} .
$$

TheOREM 2.1. Let the assumption $(\mathrm{H})$ be satisfied, the functional $h$ admit the representation $h=h_{0}-h_{1}$ with $h_{0}, h_{1} \in P F_{a b}$, the number $\lambda_{a}^{*}$ be defined by the formula (44), and the condition

$$
h_{1}(1)<\lambda_{a}^{*}
$$

hold. Let, moreover, there exist

$$
\ell_{0}, \ell_{1} \in P_{a b}
$$

such that, for any $v \in C([a, b] ; \mathbb{R})$, the inequality

$$
\left[F(v)(t)-\ell_{0}(v)(t)+\ell_{1}(v)(t)\right] \operatorname{sgn} v(t) \leq q\left(t,\|v\|_{C}\right) \quad \text { for almost all } t \in[a, b]
$$

holds, where the function $q \in K\left([a, b] \times \mathbb{R}_{+} ; \mathbb{R}_{+}\right)$satisfies the condition

$$
\lim _{x \rightarrow+\infty} \frac{1}{x} \int_{a}^{b} q(s, x) \mathrm{d} s=0 .
$$

If, in addition, either

$$
\begin{aligned}
& \left\|\ell_{0}\right\|<1-\frac{1}{\lambda_{a}^{*}} h_{1}(1)-\left(\frac{1}{\lambda_{a}^{*}}\left(h_{0}(1)-\lambda_{a}^{*}\right)\right)^{2}, \\
& \left\|\ell_{1}\right\|<2 \sqrt{1-\frac{1}{\lambda_{a}^{*}} h_{1}(1)-\left\|\ell_{0}\right\|}-\frac{1}{\lambda_{a}^{*}}\left(h_{0}(1)-\lambda_{a}^{*}\right),
\end{aligned}
$$




\section{ZDENĚK OPLUŠTIL}

or

$$
\begin{gathered}
\left\|\ell_{0}\right\| \geq 1-\frac{1}{\lambda_{a}^{*}} h_{1}(1)-\left(\frac{1}{\lambda_{a}^{*}}\left(h_{0}(1)-\lambda_{a}^{*}\right)\right)^{2}, \\
\lambda_{a}^{*}\left\|\ell_{0}\right\|+\left(h_{0}(1)-\lambda_{a}^{*}\right)\left\|\ell_{1}\right\|<\lambda_{a}^{*}-h_{1}(1)
\end{gathered}
$$

is fulfilled, then the problem (11), (2) has at least one solution.

Remark 2.1. Let $\mathcal{A}$ denote the set of $(x, y) \in \mathbb{R}_{+}^{2}$ such that either

$$
\begin{aligned}
& x<1-\frac{1}{\lambda_{a}^{*}} h_{1}(1)-\left(\frac{1}{\lambda_{a}^{*}}\left(h_{0}(1)-\lambda_{a}^{*}\right)\right)^{2}, \\
& y<2 \sqrt{1-\frac{1}{\lambda_{a}^{*}} h_{1}(1)-x}-\frac{1}{\lambda_{a}^{*}}\left(h_{0}(1)-\lambda_{a}^{*}\right),
\end{aligned}
$$

or

$$
\begin{gathered}
x \geq 1-\frac{1}{\lambda_{a}^{*}} h_{1}(1)-\left(\frac{1}{\lambda_{a}^{*}}\left(h_{0}(1)-\lambda_{a}^{*}\right)\right)^{2}, \\
\lambda_{a}^{*} x+\left(h_{0}(1)-\lambda_{a}^{*}\right) y<\lambda_{a}^{*}-h_{1}(1),
\end{gathered}
$$

where the functionals $h_{0}, h_{1} \in P F_{a b}$ are fixed and the number $\lambda_{a}^{*}$ is defined by the formula (4) (see Fig. 1). Assume that the condition (6) holds, there exist

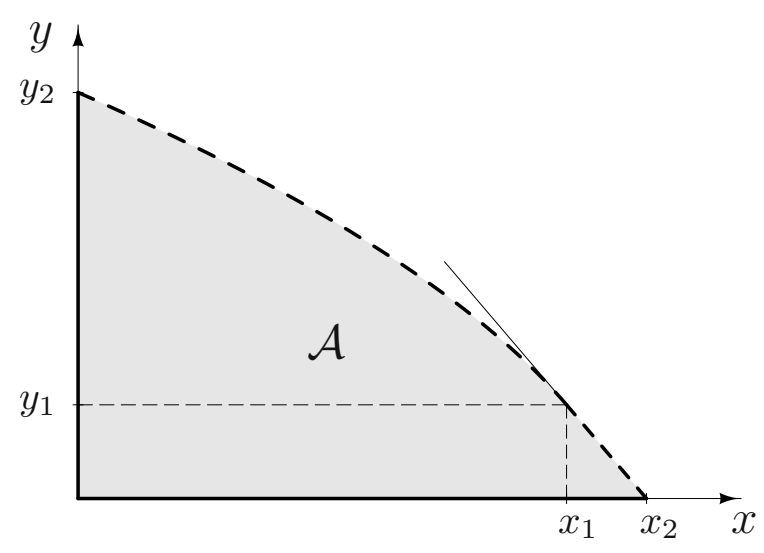

Figure 1. The set $\mathcal{A}: x_{1}=1-\frac{1}{\lambda_{a}^{*}} h_{1}(1)-\left(\frac{1}{\lambda_{a}^{*}}\left(h_{0}(1)-\lambda_{a}^{*}\right)\right)^{2}, x_{2}=1-$ $\frac{1}{\lambda_{a}^{*}} h_{1}(1), y_{1}=\frac{1}{\lambda_{a}^{*}}\left(h_{0}(1)-\lambda_{a}^{*}\right), y_{2}=2 \sqrt{1-\frac{1}{\lambda_{a}^{*}} h_{1}(1)}-\frac{1}{\lambda_{a}^{*}}\left(h_{0}(1)-\lambda_{a}^{*}\right)$.

$\ell_{0}, \ell_{1} \in P_{a b}$ such that the inequality (8) is satisfied on the set $C([a, b] ; \mathbb{R})$, and

$$
\left(\left\|\ell_{0}\right\|,\left\|\ell_{1}\right\|\right) \in \mathcal{A} \text {. }
$$

Then the problem (11), (2) with $h=h_{0}-h_{1}$ has at least one solution. 


\section{SOLVABILITY OF A LINEAR BVP FOR NONLINEAR FDES}

Remark 2.2. If the functional $h$ is defined by the formula

$$
h(v) \stackrel{\text { def }}{=} \alpha v(a)+\beta v(b) \quad \text { for } \quad v \in C([a, b] ; \mathbb{R})
$$

with $\alpha, \beta>0$, the assumptions (10) and (11) of the previous theorem take the forms

$$
\left\|\ell_{0}\right\|<1-\left(\frac{\beta}{\alpha}\right)^{2}, \quad\left\|\ell_{1}\right\|<-\frac{\beta}{\alpha}+2 \sqrt{1-\left\|\ell_{0}\right\|}
$$

and

$$
\left\|\ell_{0}\right\| \geq 1-\left(\frac{\beta}{\alpha}\right)^{2}, \quad \alpha\left\|\ell_{0}\right\|+\beta\left\|\ell_{1}\right\|<\alpha,
$$

respectively. Thus, in this case, Theorem 2.1 reduces to Theorems 14.1 and 14.6 established in [3].

TheOREM 2.2. Let the assumption $(\mathrm{H})$ be satisfied, the functional $h$ admit the representation $h=h_{0}-h_{1}$ with $h_{0}, h_{1} \in P F_{a b}$, the number $\lambda_{b}^{*}$ be defined by the formula (4), and the condition

$$
h_{1}(1)<\lambda_{b}^{*}
$$

hold. Let, moreover, there exist $\ell_{0}, \ell_{1} \in P_{a b}$ such that, for any $v \in C([a, b] ; \mathbb{R})$, the inequality

$\left[F(v)(t)-\ell_{0}(v)(t)+\ell_{1}(v)(t)\right] \operatorname{sgn} v(t) \geq-q\left(t,\|v\|_{C}\right) \quad$ for almost all $t \in[a, b]$, where the function $q \in K\left([a, b] \times \mathbb{R}_{+} ; \mathbb{R}_{+}\right)$satisfies the condition (9), holds. If, in addition, either

or

$$
\begin{aligned}
& \left\|\ell_{1}\right\|<1-\frac{1}{\lambda_{b}^{*}} h_{1}(1)-\left(\frac{1}{\lambda_{b}^{*}}\left(h_{0}(1)-\lambda_{b}^{*}\right)\right)^{2}, \\
& \left\|\ell_{0}\right\|<2 \sqrt{1-\frac{1}{\lambda_{b}^{*}} h_{1}(1)-\left\|\ell_{1}\right\|}-\frac{1}{\lambda_{b}^{*}}\left(h_{0}(1)-\lambda_{b}^{*}\right),
\end{aligned}
$$

$$
\begin{gathered}
\left\|\ell_{1}\right\| \geq 1-\frac{1}{\lambda_{b}^{*}} h_{1}(1)-\left(\frac{1}{\lambda_{b}^{*}}\left(h_{0}(1)-\lambda_{b}^{*}\right)\right)^{2}, \\
\left(h_{0}(1)-\lambda_{b}^{*}\right)\left\|\ell_{0}\right\|+\lambda_{b}^{*}\left\|\ell_{1}\right\|<\lambda_{b}^{*}-h_{1}(1)
\end{gathered}
$$

is fulfilled, then the problem (11), (2) has at least one solution.

The next theorems deal with the unique solvability of the problem (11), (2).

TheOREM 2.3. Let the assumption $(\mathrm{H})$ be satisfied, the functional $h$ admit the representation $h=h_{0}-h_{1}$ with $h_{0}, h_{1} \in P F_{a b}$, the number $\lambda_{a}^{*}$ be defined by the 


\section{ZDENĚK OPLUŠTIL}

formula (4), and the condition (6) hold. Let, moreover, there exist $\ell_{0}, \ell_{1} \in P_{a b}$ such that the inequality

$$
\begin{array}{r}
{\left[F(v)(t)-F(w)(t)-\ell_{0}(v-w)(t)+\ell_{1}(v-w)(t)\right] \operatorname{sgn}(v(t)-w(t)) \leq 0} \\
\text { for almost all } t \in[a, b]
\end{array}
$$

holds on the space $C([a, b] ; \mathbb{R})$. If, in addition, either the conditions (10), or the conditions (11) are fulfilled, then the problem (11), (2) is uniquely solvable.

TheOREm 2.4. Let the assumption $(\mathrm{H})$ be satisfied, the functional $h$ admit the representation $h=h_{0}-h_{1}$ with $h_{0}, h_{1} \in P F_{a b}$, the number $\lambda_{b}^{*}$ be defined by the formula (4), and the condition (12) hold. Let, moreover, there exist $\ell_{0}, \ell_{1} \in P_{a b}$ such that the inequality

$$
\begin{array}{r}
{\left[F(v)(t)-F(w)(t)-\ell_{0}(v-w)(t)+\ell_{1}(v-w)(t)\right] \operatorname{sgn}(v(t)-w(t)) \geq 0} \\
\text { for almost all } t \in[a, b]
\end{array}
$$

holds on the space $C([a, b] ; \mathbb{R})$. If, in addition, either the conditions (13), or the conditions (14) are fulfilled, then the problem (11), (2) is uniquely solvable.

\section{Proofs}

The main results are proved using the lemma on a priory estimate due to Kiguradze and P ํㅡa. This lemma can be formulated as follows.

Leмma 3.1 ([5, Cor. 2]). Let there exist a positive number $\rho$ and an operator $\ell \in \mathcal{L}_{a b}$ such that homogeneous problem

$$
u^{\prime}(t)=\ell(u)(t), \quad h(u)=0
$$

has only the trivial solution and, for every $\delta \in] 0,1[$, an arbitrary function $u \in$ $A C([a, b] ; \mathbb{R})$ satisfying the relations

$$
u^{\prime}(t)=\ell(u)(t)+\delta[F(u)(t)-\ell(u)(t)] \quad \text { for almost all } t \in[a, b], \quad h(u)=\delta c
$$

admits the estimate

$$
\|u\|_{C} \leq \rho .
$$

Then the problem (10), (2) has at least one solution.

Moreover, we need the following two lemmas.

Lemma 3.2. Let the operator $\omega: C([a, b] ; \mathbb{R}) \rightarrow C([a, b] ; \mathbb{R})$ be defined by the formula

$$
\omega(z)(t) \stackrel{\text { def }}{=} z(a+b-t) \quad \text { for } \quad t \in[a, b], \quad z \in C([a, b] ; \mathbb{R}) .
$$


Put

$\widehat{F}(z)(t) \stackrel{\text { def }}{=}-F(\omega(z))(a+b-t) \quad$ for almost all $t \in[a, b] \quad$ and all $z \in C([a, b] ; \mathbb{R})$ and

$$
\widehat{h}(z) \stackrel{\text { def }}{=} h(\omega(z)) \quad \text { for } \quad z \in C([a, b] ; \mathbb{R}) .
$$

Then $u$ is a solution of the problem (11), (2) if and only if the function $v=\omega(u)$ is a solution of the problem

$$
v^{\prime}(t)=\widehat{F}(v)(t), \quad \widehat{h}(v)=c .
$$

Lemma 3.3. Let the assumption $(\mathrm{H})$ be satisfied, the functional $h$ admit the representation $h=h_{0}-h_{1}$ with $h_{0}, h_{1} \in P F_{a b}$, the number $\lambda_{a}^{*}$ be defined by the formula (4), and the condition (6) hold. Let moreover, the operators $\ell_{0}, \ell_{1} \in P_{a b}$ be such that either conditions (10), or conditions (11) are fulfilled. Then there exists $r>0$ such that, for arbitrary $c^{*} \in \mathbb{R}_{+}$and $q^{*} \in L\left([a, b] ; \mathbb{R}_{+}\right)$, every function $u \in A C([a, b] ; \mathbb{R})$ satisfying the inequalities

$$
\begin{gathered}
h(u) \operatorname{sgn} u(a) \leq c^{*}, \\
{\left[u^{\prime}(t)-\ell_{0}(u)(t)+\ell_{1}(u)(t)\right] \operatorname{sgn} u(t) \leq q^{*}(t) \quad \text { for almost all } t \in[a, b]}
\end{gathered}
$$

admits the estimate

$$
\|u\|_{C} \leq r\left(c^{*}+\left\|q^{*}\right\|_{L}\right)
$$

Proof. Let $c^{*} \in \mathbb{R}_{+}, q^{*} \in L\left([a, b] ; \mathbb{R}_{+}\right)$, and $u \in A C([a, b] ; \mathbb{R})$ satisfy the conditions (19) and (20). We shall show that the estimate (21) holds, where the number $r$ depends only on $\left\|\ell_{0}\right\|,\left\|\ell_{1}\right\|, \lambda_{a}^{*}, h_{0}(1)$, and $h_{1}(1)$. It is clear that

$$
u^{\prime}(t)=\ell_{0}(u)(t)-\ell_{1}(u)(t)+\widetilde{q}(t) \quad \text { for almost all } t \in[a, b],
$$

where

$$
\widetilde{q}(t)=u^{\prime}(t)-\ell_{0}(u)(t)+\ell_{1}(u)(t) \quad \text { for almost all } t \in[a, b] .
$$

From the condition (20) we get

$$
\widetilde{q}(t) \operatorname{sgn} u(t) \leq q^{*}(t) \quad \text { for almost all } t \in[a, b] .
$$

First suppose that the function $u$ does not change its sign. Put

$$
\bar{M}=\max \{|u(t)|: t \in[a, b]\}
$$

and choose $t_{\bar{M}} \in[a, b]$ such that

$$
\left|u\left(t_{\bar{M}}\right)\right|=\bar{M} .
$$

It is clear that $\bar{M} \geq 0$ and, moreover, according to (7), (24), and (25), from (22) we obtain

$$
|u(t)|^{\prime} \leq \bar{M} \ell_{0}(1)(t)+q^{*}(t) \quad \text { for almost all } t \in[a, b] .
$$




\section{ZDENĚK OPLUŠTIL}

In view of (3), it is clear that

$$
h(u) \operatorname{sgn} u(a)=\lambda_{a}^{*}|u(a)|-h_{1}(u) \operatorname{sgn} u(a)+h_{0, a}^{\lambda_{a}^{*}}(u) \operatorname{sgn} u(a)
$$

and thus the relations (5), (19), and (25) yield

$$
|u(a)| \leq M \frac{h_{1}(1)}{\lambda_{a}^{*}}+\frac{c^{*}}{\lambda_{a}^{*}} .
$$

The integration of (27) from $a$ to $t_{\bar{M}}$, on account of (17) and (28) results in

$$
\bar{M}-\bar{M} \frac{h_{1}(1)}{\lambda_{a}^{*}}-\frac{c^{*}}{\lambda_{a}^{*}} \leq \bar{M}\left\|\ell_{0}\right\|+\left\|q^{*}\right\|_{L} .
$$

Note that the relations (10) and (11) yield that $\left\|\ell_{0}\right\|<1-\frac{h_{1}(1)}{\lambda_{a}^{*}}$. Therefore, from the last inequality we get

$$
\|u\|_{C} \leq r_{0} \max \left\{\frac{1}{\lambda_{a}^{*}}, 1\right\}\left(c^{*}+\left\|q^{*}\right\|_{L}\right),
$$

where

$$
r_{0}=\left(1-\frac{h_{1}(1)}{\lambda_{a}^{*}}-\left\|\ell_{0}\right\|\right)^{-1}>0
$$

and thus the estimate (21) holds, where $r$ is defined by the formula

$$
r=r_{0} \max \left\{\frac{1}{\lambda_{a}^{*}}, 1\right\} .
$$

Now suppose that the function $u$ changes its sign. Put

$$
M=\max \{u(t): t \in[a, b]\}, \quad m=-\min \{u(t): t \in[a, b]\}
$$

and choose $t_{M}, t_{m} \in[a, b]$ such that

$$
u\left(t_{M}\right)=M, \quad u\left(t_{m}\right)=-m .
$$

Obviously, $M>0, m>0$, and either

$$
t_{m}<t_{M},
$$

or

$$
t_{m}>t_{M}
$$

First suppose that the relation (31) holds. It is clear that there exists $\alpha_{2} \in$ ]$t_{m}, t_{M}[$ such that

$$
u(t)>0 \quad \text { for } \quad \alpha_{2}<t \leq t_{M}, \quad u\left(\alpha_{2}\right)=0 .
$$

Let

$$
\alpha_{1}=\inf \left\{t \in\left[a, t_{m}\right]: u(s)<0 \quad \text { for } \quad t \leq s \leq t_{m}\right\}
$$


Obviously,

$$
u(t)<0 \quad \text { for } \quad \alpha_{1}<t \leq t_{m} \quad \text { and } \quad u\left(\alpha_{1}\right)=0 \quad \text { if } \quad \alpha_{1}>a .
$$

In view of (3), it is clear that

$$
\lambda_{a}^{*} u(a)=h_{1}(u)-h_{0, a}^{\lambda_{a}^{*}}(u)+h(u)
$$

and thus we obtain from (5), (19), (29), and (34) that

$$
u\left(\alpha_{1}\right) \geq-m \frac{h_{1}(1)}{\lambda_{a}^{*}}-M \frac{1}{\lambda_{a}^{*}}\left(h_{0}(1)-\lambda_{a}^{*}\right)-\frac{c^{*}}{\lambda_{a}^{*}} .
$$

The integration of (22) from $\alpha_{1}$ to $t_{m}$, and from $\alpha_{2}$ to $t_{M}$, in view of (17), (20), (29), (30), and (33)-(35), yields

$$
\begin{aligned}
& m-m \frac{h_{1}(1)}{\lambda_{a}^{*}}-M \frac{1}{\lambda_{a}^{*}}\left(h_{0}(1)-\lambda_{a}^{*}\right)-\frac{c^{*}}{\lambda_{a}^{*}} \\
& \leq M \int_{\alpha_{1}}^{t_{m}} \ell_{1}(1)(s) \mathrm{d} s+m \int_{\alpha_{1}}^{t_{m}} \ell_{0}(1)(s) \mathrm{d} s+\int_{\alpha_{1}}^{t_{m}} q^{*}(s) \mathrm{d} s
\end{aligned}
$$

and

$$
M \leq M \int_{\alpha_{2}}^{t_{M}} \ell_{0}(1)(s) \mathrm{d} s+m \int_{\alpha_{2}}^{t_{M}} \ell_{1}(1)(s) \mathrm{d} s+\int_{\alpha_{2}}^{t_{M}} q^{*}(s) \mathrm{d} s .
$$

Hence, we have

$$
\begin{aligned}
m\left(1-\frac{h_{1}(1)}{\lambda_{a}^{*}}-C\right) & \leq M\left(\frac{1}{\lambda_{a}^{*}}\left(h_{0}(1)-\lambda_{a}^{*}\right)+A\right)+\left\|q^{*}\right\|_{L}+\frac{c^{*}}{\lambda_{a}^{*}}, \\
M(1-D) & \leq m B+\left\|q^{*}\right\|_{L}
\end{aligned}
$$

where

$$
A=\int_{\alpha_{1}}^{t_{m}} \ell_{1}(1)(s) \mathrm{d} s, \quad B=\int_{\alpha_{2}}^{t_{M}} \ell_{1}(1)(s) \mathrm{d} s
$$

and

$$
C=\int_{\alpha_{1}}^{t_{m}} \ell_{0}(1)(s) \mathrm{d} s, \quad D=\int_{\alpha_{2}}^{t_{M}} \ell_{0}(1)(s) \mathrm{d} s .
$$




\section{ZDENĚK OPLUŠTIL}

Due to the relations (10) and (11) we have $\left\|\ell_{0}\right\|<1-\frac{1}{\lambda_{a}^{*}} h_{1}(1)$ and thus it is clear that $C<1-\frac{h_{1}(1)}{\lambda_{a}^{*}}$ and $D<1$. Consequently, (36) implies

$$
\begin{aligned}
& m\left(1-\frac{h_{1}(1)}{\lambda_{a}^{*}}-C\right)(1-D) \\
& \quad \leq m B\left(\frac{1}{\lambda_{a}^{*}}\left(h_{0}(1)-\lambda_{a}^{*}\right)+A\right)+\left(\left\|q^{*}\right\|_{L}+\frac{c^{*}}{\lambda_{a}^{*}}\right)\left(\frac{h_{0}(1)}{\lambda_{a}^{*}}+A\right), \\
& M\left(1-\frac{h_{1}(1)}{\lambda_{a}^{*}}-C\right)(1-D) \\
& \quad \leq M B\left(\frac{h_{0}(1)}{\lambda_{a}^{*}}-1+A\right)+\left(\left\|q^{*}\right\|_{L}+\frac{c^{*}}{\lambda_{a}^{*}}\right)(B+1) .
\end{aligned}
$$

It is clear that

$$
\left(1-\frac{h_{1}(1)}{\lambda_{a}^{*}}-C\right)(1-D) \geq 1-\frac{h_{1}(1)}{\lambda_{a}^{*}}-(C+D) \geq 1-\frac{h_{1}(1)}{\lambda_{a}^{*}}-\left\|\ell_{0}\right\| .
$$

Let first the inequalities (10) hold. Obviously,

$$
B\left(\frac{h_{0}(1)}{\lambda_{a}^{*}}-1+A\right) \leq \frac{1}{4}\left(A+B+\frac{h_{0}(1)}{\lambda_{a}^{*}}-1\right)^{2} \leq \frac{1}{4}\left(\left\|\ell_{1}\right\|+\frac{h_{0}(1)}{\lambda_{a}^{*}}-1\right)^{2} .
$$

By the last inequality, (38) and the second inequality in (10), from (37) we get

where

$$
\begin{aligned}
& m \leq r_{1} \max \left\{\frac{1}{\lambda_{a}^{*}}, 1\right\}\left(\left\|q^{*}\right\|_{L}+c^{*}\right)\left(\frac{h_{0}(1)}{\lambda_{a}^{*}}+\left\|\ell_{1}\right\|\right), \\
& M \leq r_{1} \max \left\{\frac{1}{\lambda_{a}^{*}}, 1\right\}\left(\left\|q^{*}\right\|_{L}+c^{*}\right)\left(1+\left\|\ell_{1}\right\|\right),
\end{aligned}
$$

$$
r_{1}=\left[1-\frac{h_{1}(1)}{\lambda_{a}^{*}}-\left\|\ell_{0}\right\|-\frac{1}{4}\left(\left\|\ell_{1}\right\|+\frac{h_{0}(1)}{\lambda_{a}^{*}}-1\right)^{2}\right]^{-1} .
$$

Consequently, the estimate (21) holds, where the number $r$ is defined by the formula

$$
r=r_{1} \max \left\{\frac{1}{\lambda_{a}^{*}}, 1\right\}\left(\frac{h_{0}(1)}{\lambda_{a}^{*}}+\left\|\ell_{1}\right\|\right),
$$

because we have $\lambda_{a}^{*} \leq h_{0}(1)$.

Now let the inequalities (11) hold. Using the relation $\lambda_{a}^{*} \leq h_{0}(1)$, from the inequalities (11) we get

$$
B \leq\left\|\ell_{1}\right\|<\frac{h_{0}(1)}{\lambda_{a}^{*}}-1
$$


and thus

$$
B\left(\frac{h_{0}(1)}{\lambda_{a}^{*}}-1+A\right) \leq B\left(\frac{h_{0}(1)}{\lambda_{a}^{*}}-1\right)+A\left(\frac{h_{0}(1)}{\lambda_{a}^{*}}-1\right) \leq\left\|\ell_{1}\right\|\left(\frac{h_{0}(1)}{\lambda_{a}^{*}}-1\right) .
$$

By the last inequality, (38) and the second inequality in (11), it follows from (37) that

where

$$
\begin{aligned}
& m \leq r_{2} \max \left\{\frac{1}{\lambda_{a}^{*}}, 1\right\}\left(\left\|q^{*}\right\|_{L}+c^{*}\right)\left(\frac{h_{0}(1)}{\lambda_{a}^{*}}+\left\|\ell_{1}\right\|\right), \\
& M \leq r_{2} \max \left\{\frac{1}{\lambda_{a}^{*}}, 1\right\}\left(\left\|q^{*}\right\|_{L}+c^{*}\right)\left(1+\left\|\ell_{1}\right\|\right),
\end{aligned}
$$

$$
r_{2}=\left[1-\frac{h_{1}(1)}{\lambda_{a}^{*}}-\left\|\ell_{0}\right\|-\left\|\ell_{1}\right\|\left(\frac{h_{0}(1)}{\lambda_{a}^{*}}-1\right)\right]^{-1} .
$$

Consequently, the estimate (21) holds, where the number $r$ is given by the formula

$$
r=r_{2} \max \left\{\frac{1}{\lambda_{a}^{*}}, 1\right\}\left(\frac{h_{0}(1)}{\lambda_{a}^{*}}+\left\|\ell_{1}\right\|\right) .
$$

If the relation (32) holds, the validity of estimate (21) can be proved analogously.

Now we are in a position to prove Theorems 2.1 2.4

Proof of Theorem 2.1. Let $\ell=\ell_{0}-\ell_{1}$. It is clear that $\ell \in \mathcal{L}_{a b}$ and the assumptions of Lemma 3.3 are satisfied. Let $r$ be the number appearing in this lemma. According to (9), there exists $\rho>2 r|c|$ such that

$$
\frac{1}{x} \int_{a}^{b} q(s, x) \mathrm{d} s<\frac{1}{2 r} \quad \text { for } \quad x>\rho .
$$

First note that the homogeneous problem (16) has only the trivial solution. Indeed, if $u$ is a solution of the problem (16) then the function $u$ satisfies the inequalities (19) and (20) with $c^{*}=0$ and $q^{*} \equiv 0$ and thus Lemma 3.3 guarantees that $u \equiv 0$.

Now assume that a function $u \in A C([a, b] ; \mathbb{R})$ satisfies the conditions (17) for some $\delta \in] 0,1[$. Then we obtain from (8) that inequalities (19) and (20) are fulfilled with $c^{*}=|c|$ and $q^{*} \equiv q\left(\cdot,\|u\|_{C}\right)$. Hence, using Lemma 3.3 and the definition of the number $\rho$, we get the estimate (18). Indeed, assuming that $\|u\|_{C}>\rho$, from the estimate (21) we get

$$
1 \leq \frac{r|c|}{\|u\|_{C}}+\frac{r}{\|u\|_{C}} \int_{a}^{b} q\left(s,\|u\|_{C}\right) \mathrm{d} s<1
$$




\section{ZDENĚK OPLUŠTIL}

which is a contradiction.

Since $\rho$ depends neither on $u$ nor on $\delta$, it follows from Lemma 3.1 that the problem (11), (2) has at least one solution.

Proof of Theorem 2.2. According to Lemma 3.2, the validity of the theorem follows immediately from Theorem 2.1 .

Proof of Theorem 2.3. First note that the assumptions of Lemma 3.3 are satisfied.

It follows from the condition (15) that the inequality (8) is fulfilled on the set $C([a, b] ; \mathbb{R})$, where $q \equiv|F(0)|$. Consequently, all the assumptions of Theorem 2.1 are satisfied and thus the problem (11), (2) has at least one solution. It remains to show that problem (11), (2) has at most one solution.

Let $u_{1}, u_{2}$ be arbitrary solutions of the problem (11), (2). Put

$$
u(t)=u_{1}(t)-u_{2}(t) \quad \text { for } \quad t \in[a, b] .
$$

Then $h(u)=0$ and, by virtue of the inequality (15), we have

$$
\left[u^{\prime}(t)-\ell_{0}(u)(t)+\ell_{1}(u)(t)\right] \operatorname{sgn} u(t) \leq 0 \quad \text { for almost all } t \in[a, b] .
$$

Consequently, the inequalities (19) and (20) are satisfied with $c^{*}=0$ and $q^{*} \equiv 0$. Therefore, Lemma 3.3 guarantees that $u \equiv 0$ and thus $u_{1} \equiv u_{2}$.

Proof of Theorem 2.4. According to Lemma 3.2, the validity of the theorem follows immediately from Theorem 2.3 .

\section{REFERENCES}

[1] AZBELEV, N. V.-MAKSIMOV, V. P.-RAKHMATULLinA, L. F.: Introduction to the Theory of Functional Differential Equations. Nauka, Moscow, 1991. (In Russian)

[2] HAKL, R.-LOMTATIDZE, A.-ŠSEMR, J.: On an antiperiodic type boundary-value problem for first order nonlinear functional differential equations of non-Volterra's type, Nelinijni Kolyvannya 6 (2003), No. 4, 550-573.

[3] HAKL, R.-LOMTATIDZE, A.-ŠREMR, J.: Some Boundary Value Problems for First Order Scalar Functional-Differential Equations. Folia Fac. Sci. Natur. Univ. Masaryk. Brun. Math., Vol. 10, Masaryk University, Brno, 2002.

[4] HALE, J.: Theory of Functional Differential Equations (2nd ed.), Appl. Math. Sci., Vol. 3, Springer-Verlag, Berlin, 1977.

[5] KIGURADZE, I.-PÜŽA, B.: On boundary value problems for functional-differential equations, Mem. Differential Equations Math. Phys. 12 (1997), 106-113.

[6] KOLMANOVSKII, V.-MYSHKIS, A.: Introduction to the Theory and Applications of Functional Differential Equations. Math. Appl., Vol. 463, Kluwer Acad. Publ., Dordrecht, 1999.

[7] KOLMOGOROV, A. N.-FOMIN, S. V.: Elements of the Theory of Functions and Functional Analysis. Dover Publications Inc., Mineola, NY, 1999. 


\section{SOLVABILITY OF A LINEAR BVP FOR NONLINEAR FDES}

[8] SCHWABIK, Š.-TVRDÝ, M.-VEJVODA, O.: Differential and Integral Equations. Boundary Value Problems and Adjoints. D. Reidel Publ. Co., Dordrecht, Academia, Praha, 1979.

Received September 24, 2008

Institute of Mathematics

Faculty of Mechanical Engineering

Technická 2

CZ-616-69 Brno

CZECH REPUBLIC

E-mail: oplustil@fme.vutbr.cz 